\title{
Aumento na incidência de trombose venosa cerebral em região endêmica de febre amarela
}

\author{
Increase in the incidence of cerebral venous thrombosis in endemic region of yellow fever \\ Aumento de la incidencia de trombosis venosa cerebral en la región endémica de la fiebre \\ amarilla
}

Alex Nagem Machado1*, Debora Nagem Machado José ${ }^{1}$, Giane Prata da Costa Filha ${ }^{2}$, Luiza Gomes Santiago ${ }^{1}$, Yan Ker Marrara Peixoto ${ }^{1}$.

\section{RESUMO}

Objetivo: O estudo tem a proposta de investigar o evento adverso relacionado com a elevada incidência de trombose venosa cerebral em região endêmica de febre amarela. Métodos: Analisou-se a incidência de 17 casos, em um intervalo de 5 meses, com quadro clínico de hipertensão intracraniana. Os pacientes foram submetidos a propedêutica por imagem do encéfalo, revelando sinais de trombose venosa cerebral (TVC). Resultados: A avaliação epidemiológica revelou que 4 dos pacientes apresentavam fatores de risco para TVC, no entanto 4 casos manifestaram sinais clínicos e laboratoriais de meningite asséptica. A exposição direta ou indireta ao o vírus vivo atenuado da vacina de febre amarela foi observado em 16 pacientes. A investigação sorológica foi realizado em 10 pacientes. Conclusão: Os resultados sorológicos mostraram-se indetectáveis, no entanto o tempo de coleta após $8^{\circ}$ dia favorece resultados falso negativos, sendo os dados clínicos e epidemiológicos recomendados para a confirmação do diagnóstico. O tratamento indicado foi o uso de anticoagulante com recuperação total em 15 pacientes e a melhora funcional parcial observada em 2 casos.

Palavras-chave: Trombose venosa cerebral, Meningite asséptica, Febre amarela.

\begin{abstract}
Objective: The study aims to investigate the adverse event related to the high incidence of cerebral venous thrombosis in an endemic area of yellow fever. Methods: The incidence of 17 cases, over an interval of 5 months, with a clinical picture of intracranial hypertension was analyzed. The patients underwent brain imaging tests, showing signs of cerebral venous thrombosis (CVT). Results: The epidemiological evaluation revealed that 4 of the patients had risk factors for CVT, however 4 cases showed clinical and laboratory signs of aseptic meningitis. Direct or indirect exposure to the live attenuated virus of the yellow fever vaccine was observed in 16 patients. Serological investigation was carried out on 10 patients. Conclusion: The serological results were undetectable, however the collection time after the 8th day favors false negative results, and the clinical and epidemiological data are recommended to confirm the diagnosis. The indicated treatment was the use of anticoagulant with total recovery in 15 patients and the partial functional improvement observed in 2 cases.
\end{abstract}

Keywords: Intracranial venous thrombosis, Aseptic meningitis, Yellow fever.

\section{RESUMEN}

Objetivo: El estudio tiene como objetivo investigar el evento adverso relacionado con la alta incidencia de trombosis venosa cerebral en un área endémica de fiebre amarilla. Métodos: Se analizó la incidencia de 17 casos, en un intervalo de 5 meses, con un cuadro clínico de hipertensión intracraneal. Los pacientes se sometieron a pruebas de imágenes cerebrales, que muestran signos de trombosis venosa cerebral (TVC). Resultados: La evaluación epidemiológica reveló que 4 de los pacientes tenían factores de riesgo para TVC, sin embargo, 4 casos mostraron signos clínicos y de laboratorio de meningitis aséptica. Se observó exposición directa o indirecta al virus vivo atenuado de la vacuna contra la fiebre amarilla en 16 pacientes. La investigación serológica se llevó a cabo en 10 pacientes. Conclusión: Los resultados serológicos fueron indetectables, sin embargo, el tiempo de recolección después del octavo día favorece los resultados falsos negativos, y se recomiendan los datos clínicos y epidemiológicos para confirmar el diagnóstico. El tratamiento indicado fue el uso de anticoagulantes con recuperación total en 15 pacientes y la mejora funcional parcial observada en 2 casos.

Palabras clave: Trombosis venosa cerebral, Meningitis aséptica, Fiebre amarilla.

${ }^{1}$ Centro Universitário UNIFACIG, Manhuaçu - MG. *E-mail: alex.nagem@globo.com

${ }^{2}$ Centro Universitário Saúde ABC, Santo André - SP. 


\section{INTRODUÇÃO}

A trombose venosa cerebral (TVC) é uma patologia grave, no entanto pouco frequente, comprometendo a drenagem venosa do encéfalo, acarretando infarto venoso e hipertensão intracraniana, podendo evoluir com edema cerebral grave. Os dados epidemiológicos na literatura descrevem a TVC com incidência de 3 a 4 casos/100.000 habitantes/ano, segundo os estudos de correspondendo à $0,5 \%$ dos casos de acidente vascular cerebral (AVC) (CHIK Y, et al., 2012; DUTRA AP, 2008). Estudos recentes utilizando novas metodologias de investigação, descrevem uma incidência de 15,7 casos/100.000 habitante/ano, considerado a maior incidência descrita na literatura (DEVASAGAYAM S, et al., 2016).

O uso de anticonceptivo oral e a gravidez são as principais causas de TVC em adultos (COUTINHO et al., 2012). As patologias relacionadas com distúrbios de coagulação são fatores predisponentes habitualmente relacionados com a etiologia da TVC. A mutação do Fator $\mathrm{V}$ de Leiden e a resistência à proteína $\mathrm{C}$ representam 10 a $25 \%$ dos casos no entanto, outras coagulopatias podem estar relacionadas com fator de risco como: deficiência de proteína $\mathrm{C}$ e proteína $\mathrm{S}$, hiperhomocisteinemia, deficiência de antitrombina III, Síndrome do anticorpo antifosfolipídio, deficiência de plasminogênio (WEIMAR C, 2014). Causas menos frequentes de TVC incluem os distúrbios hematológicos, anemia hemolítica, colagenoses, hidrocefalia obstrutiva e comunicante, drenagem venosa prejudicada, medicamentos (quimioterápicos, eritropoetina, corticóide, vitamina A em altas doses) e distúrbios metabólicos (tireotoxicose, uremia, diabetes).

A etiologia infecciosa é considerada a causa em 18\% dos casos de TVC, geralmente relacionados com meningite, encefalite, otite, sinusite e abscesso cerebral (WEIMAR C, 2014). Os sintomas da TVC são muitas vezes inespecíficos, predominando a cefaléia que pode estar presente em $90 \%$ dos pacientes na fase aguda e $64 \%$ dos pacientes em fase subaguda (CUMURCIUC R, et al., 2005). A evolução, desde o início da fisiopatologia até o surgimento dos primeiros sintomas, varia em média de 7 dias. Outros sinais e sintomas podem ser descritos como náuseas, vômitos, papiledema, crise convulsiva, afasia, déficit motor, edema cerebral e coma (FILIPPIDIS A, et al., 2009).

Conforme a manifestação clínica, a TVC pode ser dividida em quatro grupo sindrômicos: hipertensão intracraniana isolada, alterações neurológicas focais, convulsões e encefalopatia (PIAZZA G,2012). O diagnóstico é baseado no contexto clínico associado à propedêutica de imagem por ressonância nuclear magnética do encéfalo (RNM), angioressonância do encéfalo (Angio-RNM) e angiografia digital com subtração, no entanto, estudos recentes demonstram sensibilidade superior com exames de venografia por tomografia do encéfalo (MAJOIE CB, et al., 2004; YANG Q, et al., 2016).

A meningite asséptica é um dos fatores predisponentes para TVC. As infecções por enterovírus são as principais causas de meningite asséptica, no entanto publicações demonstram a relação dos flavivírus como agente etiológico de meningite asséptica, apresentando-se de forma neuroinvasiva e evoluindo com TVC (ONDER H, 2016; VASANTHI N, et al., 2015).

O objetivo do estudo foi analisar a ocorrência de evento adverso durante a epidemia de febre amarela, observando um aumento na incidência de meningite asséptica e sua complicação com trombose venosa cerebral, em pacientes submetido a imunização com o vírus vivo atenuado da febre amarela.

\section{MÉTODOS}

O estudo foi submetido à apreciação e aprovação pelo Comitê de Ética em Pesquisas/CONEP e avaliou um grupo de 17 pacientes, de forma retrospectiva e analítica, com sintomas clínicos e radiológicos compatíveis com trombose venosa cerebral, entre janeiro de 2017 e maio de 2017. A pesquisa foi realizada no município de Manhuaçu, região leste do estado de Minas Gerais, localidade que no momento da investigação enfrentava o pior surto de febre amarela de sua história.

O estudo teve início a partir dos indícios de aumento no número de pacientes que procuravam atendimento médico com sintomas de cefaléia intensa associado a alterações neurológicas, e histórico recente de contato com o vírus vivo atenuado da febre amarela através de vacinação. Os pacientes selecionados para o estudo foram submetidos inicialmente a propedêutica de imagem por tomografia computadorizada crânio (TC) e 
posteriormente a ressonância magnética do encéfalo (RNM) e angioressonância do encéfalo (Angio-RN), revelando um elevado número de casos com alterações no fluxo sanguíneo nos seios venosos durais, sugestivos de TVC. Os casos foram notificados ao Sistema de Informação de Agravos de Notificação (SINAN) como casos suspeitos de febre amarela.

O critério de inclusão clínica foi os pacientes que apresentaram quadro de cefaléia com sinais de hipertensão intracraniana, sendo excluídos os casos de cefaléia primária e os de cefaléia secundária de etiologia não compatível com TVC. O critério de inclusão radiológico foi a identificação de ausência de fluxo nos seios venosos durais nos exames de Angio-RN, e imagens com sinais de trombos nos seios, sendo hipointensos em T1 e T2 na fase aguda, e hiperintenso em T1 e T2 na fase subaguda, além do estudo da anatomia do sulco do seio sigmóide e hipoplasia do seio transverso.

Foram analisados os dados patológicos na TC, subdivididos em sinais diretos (sinal da corda, sinal do seio transverso hiperdenso, sinal da veia jugular hiperdensa, sinal do triângulo denso no seio sagital superior) e indiretos (infarto hemorrágico, edema, colapso ventricular, hemorragia subaracnóide por infarto venoso). Os fatores predisponentes para TVC como condições pró-trombóticas genéticas e adquiridas foram avaliados.

$\mathrm{Na}$ fase inicial da pesquisa, com a investigação dos casos de cefaléia atípica, foi realizado a notificação como casos suspeitos de febre amarela. Diante da notificação dos casos em investigação e a suspeita de evento adverso relacionado com a imunização para febre amarela, foram realizadas visitas técnicas pela Subsecretaria de Vigilância e Proteção à Saúde da Secretaria Estadual de Saúde de Minas Gerais.

Os pacientes foram submetidos a investigação epidemiológica para evento adverso relacionado à imunização para febre amarela, com coleta de sangue e urina para realização de testes sorológicos como: PCR em tempo real, isolamento viral, IgM Febre amarela em sangue, plasma e urina. Foram realizados a análise de líquor em três pacientes sem contra-indicação para raquicentese propedêutica.

A hidrocefalia com hipertensão intracraniana foi observada em um paciente, sendo submetido a cirurgia de derivação ventrículo-peritoneal, e durante $o$ ato cirúrgico foi coletado fragmento de tecido de dura-máter para pesquisa viral. A amostra de leptomeninge foi adequadamente acondicionada em recipiente com crioproteção sendo enviado para a Fundação Ezequiel Dias - FUNED, para investigação e posteriormente encaminhado ao laboratório de flavivírus da Fundação Oswaldo Cruz.

\section{RESULTADOS}

Foram selecionados 17 pacientes para o estudo, com quadro clínico e radiológico compatíveis com TVC. Observou-se a ocorrência de 11 casos do sexo feminino e 6 casos do sexo masculino. A ocorrência dos principais sinais e sintomas estão descritos na tabela 1. A evolução clínica observada para TVC foi de 2 casos na fase subaguda e 15 casos na fase crônica.

A interpretação das imagens por tomografia do crânio no atendimento inicial, mostrou-se dentro dos padrões de normalidade em 13 pacientes. Os casos com anormalidades radiológicas demonstraram hidrocefalia comunicante em 2 casos e infarto venoso com transformação hemorrágica em outros 2 casos.

Analisando os fatores de risco para TVC identificamos a ocorrência de meningite asséptica em 4 casos, que foram confirmados pela análise bioquímica do líquor. O uso de anticonceptivo oral foi observado em 2 pacientes, enquanto tabagismo em 2 pacientes e cirurgia neurológica recente em 1 paciente. A ocorrência de meningite asséptica em $23 \%$ dos casos investigados foi superior aos dados observados na literatura e alertou sobre a possível relação dos casos de TVC com etiologia viral.

Realizou-se a investigação sobre os fatores de risco envolvidos na etiologia das meningites assépticas. A análise dos resultados revelou que 11 dos pacientes haviam recebido vacinação recente com vírus vivo atenuado da febre amarela, na dose de $0,5 \mathrm{ml}$. No entanto, observamos que 4 pacientes do sexo feminino não foram imunizadas com a vacina para febre amarela, entretanto informaram que os parceiros sexuais haviam sido imunizados contra febre amarela, conforme documentado nos cartões de vacinação. 
A forma de transmissão sexual foi incluída para o estudo, considerando a ocorrência de caso confirmado de transmissão vertical materno-fetal no mesmo período e região geográfica da realização do estudo. A gastroenterite viral foi outra etiologia infecciosa observada em 1 paciente, conforme descrito na (Tabela 1).

Tabela 1 - Ocorrência dos principais sinais e sintomas dos pacientes do estudo.

\begin{tabular}{cc}
\hline Sinal e sintoma & Ocorrência/total 17 pacientes \\
\hline Cefaléia & 17 \\
Náuseas e vômitos & 17 \\
Sonolência & 13 \\
Piora da visão & 7 \\
Piora da fala & 7 \\
Convulsão & 7 \\
Piora motora & 5 \\
Alterações cognitivas & 2
\end{tabular}

Fonte: Machado AN, et al.,2020.

Os pacientes foram submetidos a investigação de fatores pró-trombóticos (fator $V$ de Leidein, deficiência de antitrombina III e deficiência de proteína $C$ e proteína $S$, anticorpos antifosfolipídeos, homocisteína), fatores inflamatórios (VHS, VDRL, fator antinuclear - FAN) e fatores metabólicos (glicemia, TSH, uréia).

A investigação sorológica foi realizada sob orientação da Subsecretaria de Vigilância e Proteção à Saúde da Secretaria Estadual de Saúde de Minas Gerais, sendo as amostras coletadas com tempo médio entre início dos sintomas e a realização da coleta das amostras de 55 dias, posteriormente foram anexadas as amostras de líquor em 3 pacientes e realizado investigação para febre amarela com reação imunoenzimática de captura IgM (MAC - ELISA), isolamento viral e imunofluorescência indireta. Os pacientes submetidos a investigação de plasma, soro e urina representavam 11 dos casos.

A interpretação dos dados sorológicos revelou um caso com resultado indeterminado com reação imunoenzimática lgM para dengue e os resultados de sorologia para febre amarela mostraram-se indetectáveis. A análise do fragmento de leptomeninge, realizada pelo laboratório de enterovírus da Fundação Oswaldo Cruz revelou-se negativa para enterovírus, afastando a principal etiologia para meningite asséptica, sem descartar a possibilidade de infecção por flavivírus.

As avaliações dos dados sorológicos negativos não podem ser consideradas como definitivas para o diagnóstico em decorrência da possibilidade de resultados de falso negativo. As coletas das amostras foram realizadas em média 55 dias após o início dos sintomas, sendo a recomendação do Ministério da Saúde para a realização das coletas de amostras de soro e sangue até o $5^{\circ}$ dia, e urina até o $8^{\circ}$ dia após o início dos sintomas.

Conforme as informações clínicas e laboratoriais podemos observar que os resultados falsos negativos não excluem a possibilidade de infecção por febre amarela. Os resultados deste estudo, revelam que 13/17 $(76,4 \%)$ pacientes foram tratados com anticoagulantes, $1 / 17$ conduta expectante $(5,8 \%), 1 / 17$ foi submetido a derivação ventrículo-peritoneal $(5,8 \%)$ e 1 paciente recebeu antiagregante plaquetário $(5,8 \%)$. A decisão para a indicação de novos anticoagulantes orais como o inibidor de fator IX em 6 pacientes foi fundamentada no fato destes pacientes residirem em locais de difícil acesso e mostrarem resistência quanto a orientação de controle laboratorial periódico de coagulograma.

A evolução clínica foi satisfatória em todos os casos, observando melhora clínica em 17 (100\%) pacientes, 2 pacientes apresentavam hemiparesia residual em recuperação $(11 \%)$, ausência de complicações hemorrágicas e nenhum caso de óbito.

REAS/EJCH | Vol.12(10) | e4218 | DOI: https://doi.org/10.25248/reas.e4218.2020 Página 4 de 7 


\section{DISCUSSÃO}

A revisão dos artigos com dados em dissecções de cadáveres demonstraram a dominância do seio transverso à direita em $45-75 \%$ dos casos e a dominância para o seio transverso esquerdo em $14-29 \%$, no entanto, o estudo morfológico demonstrou a hipoplasia do seio transverso esquerdo em 3,4\% e hipoplasia do seio transverso direito em $0,8 \%$ dos casos (SHIMA T, et al., 1996). A importância do conhecimento anatômico reforça a hipótese de hipervalorização da hipoplasia de seios transversos, pois tal afirmação baseia-se no fato de que patologias do seio sagital superior (SSS) apresentam sintomatologia inespecífica, manifestando na forma clínica de alterações de cognição e comportamento, sendo habitualmente subdiagnosticada.

O conhecimento da fisiopatologia que envolve os seios venosos transversos é fundamental para a diferenciação de condições como a trombose venosa cerebral e a estenose de seio dural. O seio transverso e o SSS têm importante papel nos mecanismos de absorção de líquor, o que justifica a ocorrência de hidrocefalia comunicante em $6,6 \%$ dos casos de trombose venosa cerebral. Os sintomas de trombose venosa cerebral podem ser classificados conforme a sua evolução em aguda (os sintomas ocorrem em até 48 horas, observado em $28 \%$ a $48 \%$ dos casos); subaguda (os sintomas ocorrem entre 48 horas a 30 dias, observado em $40 \%$ a $42 \%$ dos casos); e crônica (sintomas ocorrem após 30 dias, representando $25 \%$ a $30 \%$ dos casos) (DUTRA AP, 2008).

A cefaléia é o sintoma mais comum endo observada em $90 \%$ dos pacientes na fase aguda e $64 \%$ dos casos na fase subaguda, e em alguns casos a cefaléia pode ser tão intensa, semelhante à hemorragia subaracnóide, podendo agravar-se com os mecanismos de Valsalva (CUMURCIUC R, et al., 2005). Os sintomas focais apresentam relação com o seio venoso acometido. A crise convulsiva é outro sintoma frequentemente observado, sendo decorrente da injúria cortical com comprometimento de parênquima (FERRO JM, et al., 2004).

A propedêutica por imagem na investigação de patologias obstrutivas dos seios durais, como a trombose venosa cerebral e a estenose de seio dural, habitualmente é o fator de discrepância entre muitos estudos (FARB R, et al., 2013). A apresentação clínica e radiológica da trombose venosa cerebral pode apresentarse de forma inespecífica, limitando o diagnóstico por meios de imagem (FILIPPIDIS A, et al., 2009; YANG Q, et al., 2016). O fator de maior discrepância na interpretação dos exames de imagem é o tempo de evolução do trombo e as patologias que acometem os seios transversos, com destaque para as condições de fluxo assimétrico.

A tomografia de crânio, realizada no atendimento inicial, pode revelar dados sugestivos de trombose de seio dural, entretanto sua interpretação está fundamentada no criterioso conhecimento da anatomia dos seios durais e dos entalhes ósseos na base do crânio. O exame tomográfico do crânio com contraste apresenta sinais diretos (sinal do delta vazio e ectasia venosa), e indiretos como o realce tentorial. Cabe salientar, que os sinais patognomônicos de trombose venosa cerebral será observado em somente um terço dos casos submetidos a exame de tomografia (FILIPPIDIS A, et al., 2009).

A observação de sinais de hiperdensidade isoladas de seios venosos podem ter sensibilidade de $100 \%$ e especificidade de $95 \%$, no entanto deve ser considerado a probabilidade de superestimação (ALSAFI A, et al., 2015). A interpretação radiológica das estruturas ósseas da base do crânio, analisando o sulco do seio sigmóide e suas caracteristicas morfológicas, podem ser úteis na investigação da trombose venosa cerebral. A profundidade do sulco do seio sigmóide será observada de forma mais evidente no seio transverso e seio sigmóide conforme a dominância .

As imagens por RNM e Angio-RN do encéfalo são métodos propedêuticos importantes na investigação de trombose venosa cerebral. A Angio-RN, muito utilizada para avaliação dos seios venosos cerebrais, tem demonstrado resultados discrepantes, que podem ser observados nas descrições da literatura bem como na interpretação médica. Os estudos atuais mostram o acometimento de trombos predominantemente nos seios transversos em $70 \%$ e seio sigmóide em $53 \%$ (COUTINHO JM, et al., 2012). Os trombos tardios podem ser confundidos com sangue venoso e parênquima cerebral, principalmente no seio transverso esquerdo (YANG Q, et al., 2016).

REAS/EJCH | Vol.12(10) | e4218 | DOI: https://doi.org/10.25248/reas.e4218.2020 Página 5 de 7 
Os fatores predisponentes relacionados com a trombose venosa cerebral podem ser genéticos ou adquiridos. O uso de anticonceptivo oral é observado como fator isolado em 10\% dos casos (WEIMAR C, 2014). As coagulopatias são observadas em $10 \%$ a $25 \%$ dos casos (deficiência de antitrombina III, deficiência de proteína $\mathrm{C}$ e proteína $\mathrm{S}$, síndrome de anticorpos antifosfolipídicos, mutação do fator $\mathrm{V}$ de Leiden com resistência de proteína C ativada, hiperhomocisteinemia, mutação de protrombina G20210A, deficiência de plasminogênio, disfibrinogenemia e coagulação intravascular disseminada).

As causas menos comuns de TVC são: neoplasias, distúrbios hematológicos (policitemia, doença falciforme, hemoglobinúria paroxística noturna, doenças hemolíticas imuno-mediadas, trombocitemia), distúrbios metabólicos (diabetes, tireotoxicose, uremia, síndrome nefrótica), colagenose, vasculites, e entre as causas raras estão a hipertensão intracraniana, a concussão cerebral, intervenções neurocirúrgicas e infecções, em destaque as meningites (WEIMAR C, 2014). Os flavivírus podem apresentar reações cruzadas com as células endoteliais bem como reduzir os níveis de proteína $C$, proteína $S$ e antitrombina III (TILARA $\mathrm{M}$, et al., 2014).

A incidência de meningite asséptica é de 11 a 27 casos/100.000 habitante - ano), sendo mais comum em crianças e os sintomas frequentemente encontrados são cefaléia, vômitos, fotofobia, sonolência, rigidez nucal e convulsão (CREPALDI PIS, et al., 2018). Aproximadamente $80 \%$ dos pacientes são assintomáticos e somente $1 \%$ dos casos apresentam a forma neuroinvasiva (SANTOS GPL, 2012).

Os agentes etiológicos mais comuns na meningite asséptica são os enterovírus, principalmente o echovírus 30 , entretanto inúmeros trabalhos tem relacionado a meningite asséptica como secundária à uma complicação pós-vacinação de caxumba, raiva, influenza e febre amarela (GUIMARD T, et al., 2009; MARTINS RM, et al., 2010). Diversos outros agentes etiológicos podem estar relacionados com a meningite asséptica como reação pós-vacinal, medicamentos, neoplasias e doenças sistêmicas (GARCEZ ACC, et al., 2010; FERRO JM, et al., 2004).

Os autores investigram a meningite asséptica como fator relacionado aos casos suspeitos de TVC e observaram 16/17 pacientes que apresentavam contato com a vacina de febre amarela (cepa 17D). $O$ contato direto, por imunização, foi observado em 11/17 pacientes e o contato indireto por via sexual foi relatado em 4/17 pacientes, condição esta que até o momento não há relatos descritos pela literatura científica. Os flavivírus, como o vírus da dengue, podem apresentar transmissão vertical variando de 1,6\% a 10,5\% das gestantes infectadas, bem como o vírus da chikungunya e o vírus da Zika (CALVET G, et al., 2016 ; KARIYAWASAM S, et al., 2010).

Estudos experimentais confirmaram a transmissão do vírus vacinal da febre amarela cepa 17D com a detecção de atividade viral em tecido fetal de camundongos em gestação (SILVA FCD 2009), justificando a recomendação de não imunização com a vacina de febre amarela em gestantes. Outra situação de transmissibilidade do vírus vacinal é através da amamentação (ROHR A, et al., 2007).

Os trabalhos de Camacho descrevem que $95 \%$ dos casos que receberam dose única de vacina da febre amarela, foram considerados como imunizados por até 40 anos, com a detecção de anticorpos neutralizantes (BASSI MR, et al., 2016; CAMACHO LAB, et al., 2004). Podemos observar em dados da literatura que o diagnóstico de evento neurotrópico relacionado à vacina de febre amarela foi confirmado por exames laboratoriais e alguns casos confirmados pela simples exposição do paciente em áreas com circulação do vírus antes do início dos sintomas, quando as sorologias forem negativas (ROMANO APM, et al., 2014).

\section{CONCLUSÃO}

O estudo analisou a ocorrência de evento adverso relacionado com a incidência de meningite asséptica e TVC em paciente submetidos a imunização com vírus vivo atenuado da febre amarela. $O$ presente trabalho demonstrou que mesmo com sorologias negativas deve ser considerado o risco de exame falso negativo, decorrente do momento da coleta de material para análise e o tempo de evolução da resposta imunológica. A suspeita clínica associada à exposição em área endêmica de febre amarela é um fator de risco que deverá ser considerado como agente etiológico de meningite asséptica e suas complicações como a trombose venosa cerebral. 


\section{REFERÊNCIAS}

1. ALSAFI A, et al. Cerebral venous sinus thrombosis, a nonenhanced CT diagnosis? Radiology research and practice, 2015.

2. AYANZEN RH, et al. Cerebral MR venography: normal anatomy and potential diagnostic pitfalls. American Journal of Neuroradiology, 2000; 21(1): 74-78.

3. BASSI MR, et al. Vaccination with Replication Deficient Adenovectors Encoding YF-17D Antigens Induces LongLasting Protection from Severe Yellow Fever Virus Infection in Mice. PLoS Neglected Tropical Disease, 2016; 10(2).

4. CALVET G, et al. Detection and sequencing of Zika virus from amniotic fluid of fetuses with microcephaly in Brazil: a case study. The Lancet infectious diseases, 2016; 16(6): 653-660.

5. CAMACHO LAB, et al. Immunogenicity of WHO-17D and Brazilian 17DD yellow fever vaccines: a randomized trial. Revista de Saúde Pública, 2004; 38(5): 671-678.

6. CHIK Y, et al. Differentiation of transverse sinus thrombosis from congenitally atretic cerebral transverse sinus with CT. Stroke, 2012; 43(7): 1968-1970.

7. COUTINHO JM, et al. The incidence of cerebral venous thrombosis. Stroke, 2012; 43(12): 3375-3377.

8. CREPALDI PIS, et al. Estudo epidemiológico e clínico sobre meningite em adultos no setor de emergência em São Paulo. Arquivos Médicos dos Hospitais e da Faculdade de Ciências Médicas da Santa Casa de São Paulo, 2018; 59(1): 1-6.

9. CUMURCIUC R, et al. Headache as the only neurological sign of cerebral venous thrombosis: a series of 17 cases. Journal of Neurology, Neurosurgery \& Psychiatry, 2005; 76(8): 1084-1087.

10. DEVASAGAYAM S, et al. Cerebral Venous Sinus Thrombosis Incidence Is Higher Than Previously Thought. Stroke, 2016; 47(9): 2180-2182.

11. DUTRA AP. Trombose venosa cerebral: evolução clínica e fatores prognósticos em 111 pacientes. 2008. Tese (Doutorado em Ciencias) - Departamento de Neurologia, Faculdade de Medicina da Universidade de São Paulo, São Paulo, 2008.

12. FARB RI, et al. Idiopathic intracranial hypertension: the prevalence and morphology of sinovenous stenosis. Neurology, 2003; 60(9): 1418-1424.

13. FERRO JM, et al. Prognosis of cerebral vein and dural sinus thrombosis: results of the International Study on Cerebral Vein and Dural Sinus Thrombosis (ISCVT). Stroke, 2004; 35(3): 664-670.

14. FILIPPIDIS A, et al. Cerebral venous sinus thrombosis: review of the demographics, pathophysiology, current diagnosis, and treatment. Neurosurgical Focus, 2009; 27(5): E3.

15. GARCEZ ACC, et al. Meningite asséptica e doença autoimune. Revista Médica de Minas Gerais, 2010; 20(2): supl. 1: S49-S51

16. GEISBÜSCH C, et al. Novel Factor Xa Inhibitor for the Treatment of Cerebral Venous and Sinus Thrombosis. Stroke, 2014; 45(8): 2469-2471.

17. GUIMARD T, et al. Incidence of yellow fever vaccine-associated neurotropic disease. The American journal of tropical medicine and hygiene, 2009; 81(6): 1141-1143.

18. KARIYAWASAM S e SENANAYAKE H. Dengue infections during pregnancy: case series from a tertiary care hospital in Sri Lanka. The Journal of Infection in Developing Countries, 2010; 4(11): 767-775.

19. KUMAR R. Aseptic meningitis: diagnosis and management. The Indian Journal of Pediatrics, 2005; 72(1): 57-63.

20. MAJOIE CB, et al. Multisection CT venography of the dural sinuses and cerebral veins by using matched mask bone elimination. American journal of neuroradiology, 2004; 25(5): 787-791.

21. MARTINS RM, et al. Yellow fever vaccine post-marketing surveillance in Brazil. Procedia in Vaccinology, 2010; 2(2): 178-183.

22. ONDER H. Letter Regarding Article, 'Unusual Presentation of Dengue Fever Cerebral Venous Thrombosis. Journal of Clinical and Diagnostic Research: JCDR, 2016; 10(8): TL01.

23. PIAZZA G. Cerebral venous thrombosis. Circulation, 2012; 125(13): 1704-1709.

24. ROHR A, et al. Reversibility of venous sinus obstruction in idiopathic intracranial hypertension. American Journal of Neuroradiology, 2007; 28(4): 656-659.

25. ROMANO APM, et al. Yellow Fever Outbreaks in Unvaccinated Populations, Brazil, 2008- 2009. PLoS Neglected Tropical Diseases, 2014; 8(3).

26. SANTOS GPL. Meningites e meningoencefalites assépticas: estudos de detecção e variabilidade genética de agentes etiológicos virais. 2012. Tese (Doutorado em Vigilância Sanitária) - Fundação Oswaldo Cruz, Instituto Nacional de Controle de Qualidade em Saúde, Rio de Janeiro, 2012.

27. SHIMA T, et al. Anatomical dominance of venous sinuses and jugular vein examined by intravenous digital subtraction angiography. Surgery of the Intracranial Venous System. Tokyo: Springer-Verlag, 1996.

28. SILVA FCD. Efeitos da vacinação contra febre amarela sobre a gestação em camundongos. 2010. Dissertação (Mestrado em Biologia Celular e Tecidual) - Instituto de Ciências Biomédicas, Universidade de São Paulo, São Paulo, 2009.

29. SILVA MLA, et al. Clinical and immunological insights on severe, adverse neurotropic and viscerotropic disease following 17D yellow fever vaccination. Clinical and Vaccine Immunology, 2010; 17(1): 118-126.

30. TILARA M, et al. A Case of Cerebral Venous Thrombosis in the Patient with Dengue. International Journal of Scientific and Research Publications, 2014; 4(8): 1-11.

31. VASANTHI N, et al. Unusual Presentation of Dengue Fever-Cerebral Venous Thrombosis. Journal of clinical and diagnostic research: JCDR, 2015; 9(6): OD09.

32. WEIMAR C. Diagnosis and treatment of cerebral venous and sinus thrombosis. Current neurology and neuroscience reports, 2014; 14(1): 417.

33. YANG Q, et al. Early detection and quantification of cerebral venous thrombosis by magnetic resonance black-blood thrombus imaging. Stroke, 2016; 47(2): 404-409. 